Georgian Mathematical Journal

Volume 11 (2004), Number 1, 43-48

\title{
PROJECTIVE BUNDLES ON INFINITE-DIMENSIONAL COMPLEX SPACES
}

\author{
E. BALLICO
}

\begin{abstract}
Let $V$ be a complex localizing Banach space with countable unconditional basis and $E$ a rank $r$ holomorphic vector bundle on $\mathbf{P}(V)$. Here we study the holomorphic embeddings of $\mathbf{P}(E)$ into products of projective spaces and the holomorphic line bundles on $\mathbf{P}(E)$. In particular we prove that if $r \geq 3$, then $H^{1}(\mathbf{P}(E), L)=0$ for every holomorphic line bundle $L$ on $\mathbf{P}(E)$.
\end{abstract}

2000 Mathematics Subject Classification: 32K05, 14N05.

Key words and phrases: Infinite-dimensional projective space, complex Banach manifold, holomorphic vector bundle, holomorphic line bundle, localizing Banach space, Banach space with countable unconditional basis.

\section{InTRODUCTION}

For any complex vector space let $\mathbf{P}(V)$ be the projective space of all onedimensional linear subspaces of $V$. Unless otherwise stated all cohomology groups of sheaves are sheaf cohomology groups or, equivalently because $\mathbf{P}(V)$ is metrizable and hence paracompact if $V$ is a Banach space, Cech cohomology groups. In this paper we prove the following geometrical properties of the complex manifold $\mathbf{P}(E)$. For the notion of localizing complex manifold, see [9], p. 509; we just note that $V$ is localizing if and only if $\mathbf{P}(V)$ is localizing.

Theorem 1. Fix an integer $r \geq 2$. Let $V$ be a localizing Banach space with countable unconditional basis and $E$ a rank $r$ holomorphic vector bundle on $\mathbf{P}(V)$. Set $X:=\mathbf{P}(E)$ and let $\pi: X \rightarrow \mathbf{P}(V)$ be the projection. Call $\mathbf{O}(1)$ the tautological line bundle on $X$ with degree one on each fiber, i.e., the only line bundle on $X$ such that $\pi_{*}(\mathbf{O}(1)) \cong E$. We have $\operatorname{Pic}(X) \cong \mathbf{Z}^{\oplus 2}$ and we may take $\pi^{*}\left(\mathcal{O}_{\mathbf{P}(V)}(1)\right)$ and $\mathbf{O}(1)$ as a basis of $\mathrm{Pic}(X)$. If $r \geq 3$ we have $H^{1}(X, L)=0$ for every $L \in \operatorname{Pic}(X)$. If $r=2$ we have $H^{1}(X, L)=0$ for every $L \in \operatorname{Pic}(X)$ such that the degree of $L$ with respect to the fibration $\pi$ is at least -1 .

Theorem 2. Fix integers $r \geq 2, s>0, n \geq 0$, infinite dimensional Banach spaces $V, V_{1}, \ldots, V_{s}$ and a rank $r$ vector bundle $E$ on $\mathbf{P}(V)$. Assume that $V$ is localizing and with countable unconditional basis. Then:

(a) If $E ¥ \mathcal{O}_{\mathbf{P}(V)}(t)^{\oplus r}$ for any integer $t$ there is no closed embedding $j$ : $\mathbf{P}(E) \rightarrow \mathbf{P}\left(V_{1}\right) \times \cdots \times \mathbf{P}\left(V_{s}\right) \times \mathbf{P}^{n}$ such that $j(\mathbf{P}(E))$ has finite codimension.

(b) Let $j: \mathbf{P}(E) \rightarrow \mathbf{P}\left(V_{1}\right) \times \cdots \times \mathbf{P}\left(V_{s}\right) \times \mathbf{P}^{n}$ be a closed embedding such that $j(\mathbf{P}(E))$ has finite codimension. Then $E \cong \mathcal{O}_{\mathbf{P}(V)}(t)^{\oplus r}$ for some 
integer $t$ (i.e., $\left.\mathbf{P}(E) \cong \mathbf{P}(V) \times \mathbf{P}^{r-1}\right), s=1, n \geq r-1, V_{1} \cong V \oplus \mathbf{C}^{x}$ for some integer $x \geq 0$ and $j$ embeds linearly each slice $\mathbf{P}(V) \times\{P\}$, $P \in \mathbf{P}^{r-1}$, as a codimension $x$ closed linear subspace of $\mathbf{P}\left(V_{1}\right) \times\{Q\}$ for some $Q \in \mathbf{P}^{n}$.

Remark 1. Take $V$ and $E$ as in the statement of Theorem 2 with $E \nsubseteq$ $\mathcal{O}_{\mathbf{P}(V)}(t)^{\oplus r}$ for any integer $t$. By part (a) of Theorem 2 the " algebraic manifold $" \mathbf{P}(E)$ cannot be embedded as a finite codimensional closed analytic subset in any finite product of projective spaces.

\section{The Proofs}

Lemma 1. Let $V$ be a Banach space. Then $H^{2}(\mathbf{P}(V), \mathbf{Z}) \cong \mathbf{Z}$ and any hyperplane of $\mathbf{P}(V)$ induces a generator of $H^{2}(\mathbf{P}(V), \mathbf{Z})$.

Proof. Since the result is well-known when $V$ has finite dimension, we may assume that $V$ is infinite-dimensional. By [13], Proposition 1.2, all cohomology groups of $V \backslash\{0\}$ vanishes. We point out that for several infinite-dimensional Banach spaces (e.g., if $V$ is a Hilbert space) $V \backslash\{0\}$ is homeomorphic to $V$ ([7]) and even diffeomorphic to it ([4] or see [8], p. 21); every separable Banach space is homeomorphic to the separable Hilbert space; it easily follows that any separable Banach space $V$ is homeomorphic to $V \backslash\{0\}$ and hence $V \backslash\{0\}$ is contractible. There is a locally trivial fiber bundle $m: V \backslash\{0\} \rightarrow \mathbf{P}(V)$ with $\mathbf{C}^{*}$ as fiber. Since $H^{2}(V \backslash\{0\}, \mathbf{Z})=0$, the Leray spectral sequence of $m$ gives the existence of an injective homomorphism $H^{2}(\mathbf{P}(V), \mathbf{Z}) \rightarrow H^{1}\left(\mathbf{C}^{*}, \mathbf{Z}\right) \cong \mathbf{Z}$. Hence to check the lemma it is sufficient to show that the class $a \in H^{2}(\mathbf{P}(V), \mathbf{Z})$ represented by a hyperplane $H$ is not torsion. Take a line $D \subset \mathbf{P}(V)$ not contained in $H$. Thus $H \cap D$ is a point, $P$. By the controvariance of cohomology groups the class $a$ induces a non-zero multiple of the class $b$ represented by $P$ in $H^{2}\left(D, \mathbf{Z}\right.$. Since $H^{2}(D, \mathbf{Z}) \cong \mathbf{Z}$ and $b$ is a generator of $H^{2}(D, \mathbf{Z}), b$ is not torsion.

Lemma 2. Let $U$ be an open subset of a Banach space and $m, k$ non-negative integers with $m+k>0$. Let $f$ be a holomorphic function on $U \times\left(\mathbf{C}^{*}\right)^{k} \times \mathbf{C}^{m}$. Then there is a Laurent expansion

$$
f=\sum_{i_{1} \in \mathbf{Z}, \ldots, i_{k} \in \mathbf{Z}, j_{1} \geq 0, \ldots j_{m} \geq 0} a_{i_{1}, \ldots, i_{k}, j_{1}, \ldots, j_{m}} z_{1}^{i_{1}} \ldots z_{k}^{i_{k}} z_{k+1}^{j_{1}} \ldots z_{k+m}^{j_{m}}
$$

in which each $a_{i_{1}, \ldots, i_{k}, j_{1}, \ldots, j_{m}}$ is a holomorphic function on $U$. For all subsets $A, B$ of $\{1, \ldots, k\}$ let $\sum_{A(\geq 0), B(<0)}[f]$ be the formal expansion given by (1) in which every index $i \in A$ varies only on the set of all non-negative integers and in which every index $i \in B$ varies only on the set of all negative integers. Then the expansion $\sum_{A(>0), B(<0)}[f]$ defines a holomorphic function on the product of $U, \mathbf{C}^{*}$ for every $i \in\{1, \ldots, k\} \backslash(A \cup B), \mathbf{C}$ for every $i \in A$ and $\mathbf{P}^{1} \backslash\{0\}$ for every $i \in B$.

Proof. It is sufficient to use the classical proof by integration of the Laurent expansion in finitely many variables, just as done in [12], 45-52, for the Cauchy 
formula. Just to simplify the notation we will only write down the case $k=1$ and $m=0$. Fix $a \in \mathbf{C}^{*}$ and integrate $f(w, z)$ with respect to the variable $z$ in the union of a circle centered at 0 and with large radius going counterclockwise and a circle with center at 0 and small radius going clockwise.

Remark 2. Let $V$ be a localizing Banach space. By [1], Remark 5, for every integer $t$ we have $H^{1}\left(\mathbf{P}(V), \mathcal{O}_{\mathbf{P}(V)}(t)\right)=0$ (sheaf cohomology).

Remark 3. A Banach space $V$ has countable unconditional basis if and only if $V \oplus \mathbf{C}$ has countable unconditional basis.

Unfortunately, since in Remark 2 we are able to handle only the first cohomology group, in this paper we will use only the case $q=1$ of the following result.

Lemma 3. Let $M$ be a complex manifold locally modelled over open subsets of a Banach space with countable unconditional basis and $E$ a rank $r$ holomorphic vector bundle on $M$. Let $f: \mathbf{P}(E) \rightarrow M$ be the projection. Then $R^{q} f_{*}(\mathbf{O}(t))=0$ for every pair $(q, t)$ of integers such that either $1 \leq q \leq r-2$ or $q \geq$ r or $q=r-1$ and $t \geq-r+1$.

Proof. It is sufficient to prove that for every pseudoconvex open subset $U$ of a Banach space with countable unconditional basis and every such pair $(q, t)$ we have $H^{q}\left(U \times \mathbf{P}^{r-1}, \pi_{2}^{*}\left(\mathcal{O}_{\mathbf{P}^{r-1}}(t)\right)\right)=0$, where $\pi_{2}: U \times \mathbf{P}^{r-1} \rightarrow \mathbf{P}^{r-1}$ is the projection. We may find a finite open covering $\mathfrak{U}$ of $U \times \mathbf{P}^{r-1}$ such that any finite intersection of open subsets of this covering is isomorphic to $U \times\left(\mathbf{C}^{*}\right)^{k} \times \mathbf{C}^{m}$ for some non-negative integers $k$ and $m$ with $k+m=r-1$. By Remark 3 for all non-negative integers $k, m$ the manifold $U \times\left(\mathbf{C}^{*}\right)^{k} \times \mathbf{C}^{m}$ is a pseudoconvex open subset of a Banach space with countable unconditional basis. Thus $H^{i}(U \times$ $\left.\left(\mathbf{C}^{*}\right)^{k} \times \mathbf{C}^{m}, \mathcal{O}_{U \times\left(\mathbf{C}^{*}\right)^{k} \times \mathbf{C}^{m}}\right)=0$ for every $i>0([10]$, Theorem 0.1$)$. Hence $\mathfrak{U}$ is a Leray covering which computes $H^{q}\left(U \times \mathbf{P}^{r-1}, \pi_{2}^{*}\left(\mathcal{O}_{\mathbf{P}^{r-1}}(t)\right)\right)$. By Lemma 2 we have the Laurent expansions which allow us to copy word for word the proof for the case $U$ a point given in [5], pp. 51-55.

Remark 4. In the set-up of Lemma 3 we only used that $M$ has a basis $\mathfrak{U}$ of open subsets such that $H^{i}\left(U, \mathcal{O}_{U}\right)=0$ for every $i>0$ and every $U \in \mathfrak{U}$. Hence by [11] we may apply Lemma 3 to complex manifolds modelled over certain Fréchet spaces.

Remark 5. Let $M$ be a complex Banach manifold and $E$ a rank $r$ holomorphic vector bundle on $M$. Let $f: \mathbf{P}(E) \rightarrow M$ be the projection and $\mathbf{O}(1)$ the tautological line bundle on $\mathbf{P}(E)$ whose restriction to any fiber of $f$ has degree one. For every holomorphic vector bundle $A$ on $M$ and all integers $i, t$ with $i \geq 0$ we have $R^{i} f_{*}\left(\mathbf{O}(t) \otimes f^{*}(A)\right) \cong A \otimes R^{i} f_{*}(\mathbf{O}(t))$. If $t<0$ we have $f_{*}(\mathbf{O}(t))=0$. We have $f_{*}\left(\mathcal{O}_{\mathbf{P}(E)}\right) \cong \mathcal{O}_{M}$. If $t>0$ we have $f_{*}(\mathbf{O}(t)) \cong S^{t}(E)$, where $S^{t}$ denotes the symmetric product. Now assume $t \geq 0, M=\mathbf{P}(V)$ and $E$ isomorphic to a direct sum of $r$ line bundles. Then $S^{t}(E)$ is a direct sum of line bundles.

Proof of Theorem 1. By Lemma 3 and Remark 5 we have $\pi_{*}\left(\mathcal{O}_{X}\right) \cong \mathcal{O}_{\mathbf{P}(V)}$ and $R^{1} \pi_{*}\left(\mathcal{O}_{X}\right)=0$. Hence from the Leray spectral sequence of $\pi$ we obtain 
$H^{1}\left(X, \mathcal{O}_{X}\right)=H^{1}\left(\mathbf{P}(V), \mathcal{O}_{\mathbf{P}(V)}\right)$. The latter sheaf cohomology group vanishes by [9] and [1], Remark 5. From the exponential sequence

$$
0 \rightarrow \mathbf{Z} \rightarrow \mathcal{O}_{X} \rightarrow \mathcal{O}_{X}^{*} \rightarrow 0
$$

we obtain an inclusion $j: \operatorname{Pic}(X) \rightarrow H^{2}(X, \mathbf{Z})$. The Leray spectral sequence of $\pi$ gives $H^{2}(X, \mathbf{Z}) \cong \mathbf{Z}^{\oplus 2}$ and that $H^{2}(X, \mathbf{Z})$ has as generators the first Chern classes of $\pi^{*}\left(\mathcal{O}_{\mathbf{P}(V)}(1)\right)$ and of $\mathbf{O}(1)$. This implies the surjectivity of $j$, proving the first assertion of Theorem 1 . All the vanishing results in the statement of Theorem 1 follow from Lemma 3, Remark 5 and the vanishing theorems proved in [9] (see [1], Remark 5).

Remark 6 . Let $T$ be a reduced and irreducible finite-dimensional complex space. For every integer $m>2(\operatorname{dim}(T))$ every holomorphic map $g: \mathbf{P}^{m} \rightarrow T$ is constant because any two fibers of $f$ are disjoint and for any two closed subvarieties $A, B$ of $\mathbf{P}^{m}$ with $\operatorname{codim}(A) \leq \operatorname{dim}(T)$ and $\operatorname{codim}(B) \leq \operatorname{dim}(T)$ we have $A \cap B \neq \varnothing$. Thus every holomorphic map $\mathbf{P}(V) \rightarrow T$ is constant if $V$ is infinite-dimensional.

Proof of Theorem 2. We have $E \cong \mathcal{O}_{\mathbf{P}(V)}\left(a_{1}\right) \oplus \cdots \oplus \mathcal{O}_{\mathbf{P}(V)}\left(a_{r}\right)$ for some integers $a_{1} \geq \cdots \geq a_{r}([9]$, Theorems 7.1 and 8.5). Set $X:=\mathbf{P}(E)$ and let $f: X \rightarrow \mathbf{P}(V)$ be the projection. Assume the existence of a closed embedding $j: X \rightarrow \mathbf{P}\left(V_{1}\right) \times$ $\cdots \times \mathbf{P}\left(V_{s}\right) \times \mathbf{P}^{n}$ with $j(X)$ of finite codimension. A section of $f$ is given by a pair $(L, u)$, where $L \in \operatorname{Pic}(\mathbf{P}(V))$ and $u: L \rightarrow E$ is a nowhere vanishing inclusion, i.e., an injection as a map of holomorphic bundles, i.e., a map whose dual map $E^{*} \rightarrow L^{*}$ is surjective. The image of a section of $f$ is biholomorphic to $\mathbf{P}(V)$. Hence by Remark 6 we see that either $n=0$ or the image of any such section is mapped to a point by the projection $\mu_{s+1}: \mathbf{P}\left(V_{1}\right) \times \cdots \times \mathbf{P}\left(V_{s}\right) \times \mathbf{P}^{n} \rightarrow \mathbf{P}^{n}$. Now we use that $E \cong \mathcal{O}_{\mathbf{P}(V)}\left(a_{1}\right) \oplus \cdots \oplus \mathcal{O}_{\mathbf{P}(V)}\left(a_{r}\right)$. Hence taking $L=\mathcal{O}_{\mathbf{P}(V)}\left(a_{r}\right)$ we see that for every $P \in X$ there is a section of $f$ whose image contains $P$. Fix any section $u$ of $f$. We have $\operatorname{codim}((j \circ u)(\mathbf{P}(V)))=\operatorname{codim}(j(X))+r-1$ and hence $j \circ u$ is a finite-codimensional embedding of $\mathbf{P}(V)$ into $\mathbf{P}\left(V_{1}\right) \times \cdots \times \mathbf{P}\left(V_{s}\right) \times \mathbf{P}^{n}$. Let $\mu_{i}: \mathbf{P}\left(V_{1}\right) \times \cdots \times \mathbf{P}\left(V_{s}\right) \times \mathbf{P}^{n} \rightarrow \mathbf{P}\left(V_{i}\right), 1 \leq i \leq s$, be the projections. As in Lemma 1 and in the proof of Theorem 1 we obtain $H^{2}\left(\mathbf{P}\left(V_{1}\right) \times \cdots \times\right.$ $\left.\mathbf{P}\left(V_{s}\right) \times \mathbf{P}^{n}, \mathbf{Z}\right) \cong \mathbf{Z}^{(s+1)}$ and $\operatorname{Pic}\left(\mathbf{P}\left(V_{1}\right) \times \cdots \times \mathbf{P}\left(V_{s}\right) \times \mathbf{P}^{n}\right) \cong \mathbf{Z}^{(s+1)}$ and that both Abelian groups are freely generated by the pull-backs of the classes of the hyperplanes of each of the factors of $\mathbf{P}\left(V_{1}\right) \times \cdots \times \mathbf{P}\left(V_{s}\right) \times \mathbf{P}^{n}$. Since $\operatorname{Pic}(\mathbf{P}(V))$ is freely generated by $\mathcal{O}_{\mathbf{P}(V)}(1)$, there are integers $x_{i}, 1 \leq i \leq s+1$, such that $\left(\mu_{i} \circ j \circ u\right)^{*}\left(\mathcal{O}_{\mathbf{P}\left(V_{i}\right)}(1)\right) \cong \mathcal{O}_{\mathbf{P}(V)}\left(x_{i}\right)$ for $1 \leq i \leq s$ and $\left(\mu_{s+1} \circ j \circ u\right)^{*}\left(\mathcal{O}_{\mathbf{P}^{n}}(1)\right) \cong$ $\mathcal{O}_{\mathbf{P}(V)}\left(x_{s+1}\right)$. We have $x_{i} \geq 0$ for every $i$ and $x_{j}=0$ if and only if $\mu_{j} \circ j \circ u$ is a point.

(i) First assume $s \geq 2$ and fix $Q \in \mathbf{P}\left(V_{2}\right) \times \cdots \times \mathbf{P}\left(V_{s}\right) \times \mathbf{P}^{n}$. The analytic set $(j \circ u)(\mathbf{P}(V)) \cap \mathbf{P}\left(V_{1}\right) \times\{Q\}$ is closed and of finite codimension in the infinite-dimensional projective space $\mathbf{P}\left(V_{1}\right) \times\{Q\}$. Hence it contains many lines, $D$ (see e.g., [2] or [3]). Since $D$ maps to $\{Q\}$ by the projection $\rho: \mathbf{P}\left(V_{1}\right) \times \cdots \times \mathbf{P}\left(V_{s}\right) \times \mathbf{P}^{n} \rightarrow \mathbf{P}\left(V_{2}\right) \times \cdots \times \mathbf{P}\left(V_{s}\right) \times \mathbf{P}^{n}$ we obtain $\operatorname{deg}(D)=$ $x_{1} \operatorname{deg}\left((j \circ u)^{-1}(D)\right.$. Since the compact Riemann Surface $(j \circ u)^{-1}(D)$ has positive 
degree, while $D$ is a line, we obtain $x_{1}=1$ and that $(j \circ u)^{-1}(D)$ has degree one, i.e., it is a line. Fix $P \in(j \circ u)^{-1}(D)$ and move the line $(j \circ u)^{-1}(D)$ among the lines of $\mathbf{P}(V)$ through $P$. For any such nearby line $R$ the set $\rho \circ j \circ u(R)$ is a point. Since $P \in R$, we get $\rho \circ j \circ u(R)=\{Q\}$ for any such $R$. Since any point of $\mathbf{P}(V)$ is contained in a line containing $P$, we obtain $\rho \circ j \circ u(\mathbf{P}(V))=\{Q\}$, i.e., $j \circ u(\mathbf{P}(V)) \subseteq \mathbf{P}\left(V_{1}\right) \times\{Q\}$. Since $V_{2}$ has infinite dimension, $j \circ u(\mathbf{P}(V))$ has not finite codimension, contradiction.

(ii) By part (i) we may assume $s=1$. First assume $s=1$ and $n=0$. By [3], Theorem 3, we obtain that for any section $u$ of $f$ the closed analytic subset $j(u(X))$ is a closed finite codimensional linear subspace of $\mathbf{P}\left(V_{1}\right)$. Let $\alpha$ and $\beta$ be the integers such that $j^{*}\left(\mathcal{O}_{\mathbf{P}\left(V_{1}\right)}(1)\right) \cong \mathbf{O}(\alpha) \otimes f^{*}\left(\mathcal{O}_{\mathbf{P}(V)}(\beta)\right)$. Since the restriction of $\mathcal{O}_{\mathbf{P}\left(V_{1}\right)}(1)$ to any compact Riemann Surface contained in $\mathbf{P}\left(V_{1}\right)$ has positive degree, we have $\alpha>0$ and $\beta>0$. First assume $a_{1}=a_{r}$. Hence there are several sections $u, v$ of $f$ such that $u(\mathbf{P}(V)) \cap v(\mathbf{P}(V))=\varnothing$. Since $j(u(\mathbf{P}(V)))$ and $j(v(\mathbf{P}(V)))$ are finite codimensional linear subspaces of $\mathbf{P}\left(V_{1}\right)$ and $V_{1}$ has infinite dimension, then $j(u(\mathbf{P}(V))) \cap j(v(\mathbf{P}(V))) \neq \varnothing$, contradicting the injectivity of $j$. Now assume $a_{1}>a_{r}$. There are nowhere vanishing morphisms $\mathcal{O}_{\mathbf{P}(V)}\left(a_{1}\right) \rightarrow E$ and $\mathcal{O}_{\mathbf{P}(V)}\left(a_{r}\right) \rightarrow E$ such that the images of the corresponding sections are disjoint. Again, we just obtained a contradiction. Now assume $s=1$ and $n>0$. First assume $a_{1}>a_{r}$. In this case we obtain a family $\left\{u_{\alpha}\right\}$ of sections of $f$ whose images $\left\{u_{\alpha}(\mathbf{P}(V))\right\}$ covers $X$ and whose images are mutually intersecting. Since each $\mu\left(u_{\alpha}(\mathbf{P}(V))\right)$ is just a point (Remark 5), we obtain the existence of $P \in \mathbf{P}^{n}$ such that $j(X) \subseteq \mathbf{P}(V) \times\{P\}$. The case $s=1$ and $n=0$ gives a contradiction. Now assume $a_{1}=a_{r}$, i.e., assume $E \cong \mathcal{O}_{\mathbf{P}(V)}\left(a_{1}\right)^{\oplus r}$. Let $\alpha^{\prime}, \beta^{\prime}$ be the integers such that $j^{*}\left(\mu_{1}^{*}\left(\mathcal{O}_{\mathbf{P}\left(V_{1}\right)}(1)\right) \otimes \mu_{2}^{*}\left(\mathcal{O}_{\mathbf{P}^{n}}(1)\right)\right) \cong \mathbf{O}\left(\alpha^{\prime}\right) \otimes f^{*}\left(\mathcal{O}_{\mathbf{P}(V)}\left(\beta^{\prime}\right)\right)$. The proof just given for the case $a_{1}>a_{r}$ shows that $\alpha^{\prime}=1$. As in the proofs in [2] or [3] (i.e., just a use of Remark 6) we see that $j$ is obtained as a Segre product of a linear embedding of $\mathbf{P}(V)$ as a finite codimensional linear subspace of $\mathbf{P}\left(V_{1}\right)$ with an embedding of $\mathbf{P}^{r-1}$ into $\mathbf{P}^{n}$, proving the theorem with a more precise form of part (b).

\section{ACKNOWLEDGEMENT}

The author was partially supported by MURST and GNSAGA of INdAM (Italy).

\section{REFERENCES}

1. E. BAllico, Branched coverings and minimal free resolution for infinite-dimensional complex spaces. Georgian Math. J. 10(2003), No. 1, 37-43.

2. E. BAllico, Analytic subsets of products of infinite-dimensional projective spaces. Georgian Math. J. 10(2003), No. 4, 603-606.

3. E. Ballico, Hypersurfaces of infinite dimensional Banach spaces, Bertini theorems and embeddings of projective spaces. Portugal. Math. 60(2003), No. 3, 373-377.

4. C. Bessaga, Every infinite-dimensional Hilbert space is diffeomorphic with its unit sphere. Bull. Acad. Polon. Sci. Sér. Sci. Math. Astronom. Phys. 14(1966), 27-31. 
5. P. GRIFfiths, Topics in algebraic and analytic geometry. Written and revised by John Adams. Mathematical Notes, No. 13. Princeton University Press, Princeton, N.J.; University of Tokyo Press, Tokyo, 1974.

6. M. I. KADEC, A proof of the topological equivalence of all separable infinite-dimensional Banach spaces. (Russian) Funkcional. Anal. i Priložen. 1(1967), 61-70.

7. V. L. KleE, JR., Convex bodies and periodic homeomorphisms in Hilbert space. Trans. Amer. Math. Soc. 74(1953), 10-43.

8. N. Kuiper, Variétés hilbertiennes: aspects géométriques. Séminaire de Mathématiques Supérieures, No. 38 (Été, 1969). Les Presses de l'Université de Montréal, Montreal, Que., 1971.

9. L. Lempert, The Dolbeault complex in infinite dimensions. I. J. Amer. Math. Soc. 11(1998), No. 3, 485-520.

10. L. Lempert, The Dolbeault complex in infinite dimensions. III. Sheaf cohomology in Banach spaces. Invent. Math. 142(2000), No. 3, 579-603.

11. L. Lempert, Analytic cohomology in Fréchet spaces. Comm. Anal. Geom. 11(2003), no. $1,17-32$.

12. J. MujicA, Complex analysis in Banach spaces. Holomorphic functions and domains of holomorphy in finite and infinite dimensions. North-Holland Mathematics Studies, 120. Notas de Matemática [Mathematical Notes], 107. North-Holland Publishing Co., Amsterdam, 1986.

13. G. Ruget, A propos des cycles analytiques de dimension infinie. Invent. Math. 8(1969), 267-312.

(Received 24.04.2003; revised 17.10.2003)

Author's address:

Department of Mathematics

University of Trento

38050 Povo (TN)

Italy

E-mail: ballico@science.unitn.it 